\title{
The effect of foregrounding on readers' use of predictive inferences
}

\author{
PAUL WHITNEY, BILL G. RITCHIE, and ROBERT S. CRANE \\ Washington State University, Pullman, Washington
}

\begin{abstract}
This research extends previous attempts to determine whether subjects make predictive inferences during comprehension. For example, when subjects read a passage about someone falling out of a 14th-story window, do they infer that the person is dead? Previous research in which lexical decision, word naming, and recognition tasks have been used for detecting predictive inferences has had mixed results. In experiment 1 , a word-stem completion task was used to test for predictive inferences. The word stems were formed from target inferences that followed either priming or control passages. The data revealed that predictive inferences are generated only about concepts that are foregrounded in the passages. In Experiments 2 and 3, lexical decision and naming were used to test for predictive inferences. The lexical decision data replicated the word-stem completion data. A control experiment ruled out a simple context-checking explanation for the lexical decision results. The naming data indicated that this tasks was not sensitive to elaborative inference generation. The results show that readers make predictive inferences, but do so selectively.
\end{abstract}

In the early 1970 s, research on how people read began to focus on the nature of the mental representation formed during comprehension. One idea that came from this research was that a passage is encoded as a mental representation that has been constructed from preexisting and text-based information (see, e.g., Bransford \& Johnson, 1973; Johnson-Laird, 1983).

A key assumption of this constructive view is that information not explicitly stated is inferred during comprehension and becomes part of the text representation. However, the evidence is mixed with respect to whether such elaborative inferences are made on-line, and theoretical positions on the extensiveness of elaboration have become quite polarized (Whitney, 1987). For example, Schank (1978) argued strongly for the view that extensive elaboration is a normal part of comprehension: "Understanding is expectation based. It is only when the expectations are useless or wrong that bottom-up processing begins" (p. 94).

In support of this view, a number of early studies yielded evidence for extensive elaboration in reading (e.g., Anderson et al., 1976; Paris \& Lindauer, 1976). In these studies, however, researchers relied on cued recall to test for use of elaborative inferences. It has become clear that

This research was supported by Air Force Office of Scientific Research Grant 91-0068 to the first author. The views expressed herein are those of the authors and should not be interpreted as necessarily representing the official policies or endorsements, either expressed or implied, or the AFOSR or the U.S. government. Experiment I formed the basis of a thesis submitted by the second author in partial fulfillment of the requirements for the MS degree. The authors thank Donna Lomen for her help with data collection. Address correspondence to Paul Whitney, Department of Psychology, Washington State University, Pullman, WA 99164-4820. cue effectiveness can represent processing that takes place at retrieval rather than at encoding (see, e.g., Corbett \& Dosher, 1978). In more recent studies, on-line priming tasks have been used to investigate inference use (e.g., Dosher \& Corbett, 1982; Potts, Keenan, \& Golding, 1988). In reviewing this literature, Rayner and Pollatsek (1989) arrived at a conclusion quite different from that of Schank (1978): "Instead, readers appear to make inferences only when they have to: that is, when they have to decide how the current text ties in with what has come before" (p. 294).

Such a strong conclusion may be premature, however; in several studies, methods other than cued recall have resulted in evidence for elaboration (e.g., Graesser \& Clark, 1985; Seifert, Robertson, \& Black, 1985; Whitney, 1986).

The conflicting evidence on elaboration appears to have arisen for two reasons. First, researchers have often investigated the question of elaboration as though it were a simple question of whether or not the inferences occur on-line. A more appropriate approach to the issue would be to determine the contexts in which readers use elaborative inferences (McKoon \& Ratcliff, 1989b; Seifert et al., 1985; Whitney \& Williams-Whitney, 1990). Second, evidence of readers' use of elaborative inferences has varied, depending on the methodology chosen to detect inferences. In the early investigations done with cued recall, readers' use of elaborative inferences was overestimated. In some recent investigations, however, researchers have used methods and materials that may work against finding the conditions in which elaborative inferences are generated.

There are, of course, many different types of elaborative inferences. In the present study, we focused on inferences in which the reader predicts the likely conse- 
quence of an action. For example, if the one reads, "The actress fell from the 14th-story window," one can probably assume that the actress died. These predictive inferences are a representative case in the general controversy over elaborations, and they have received considerable attention in the literature (e.g., Duffy, 1986; McKoon \& Ratcliff, 1986; Potts et al., 1988; Singer \& Ferreira, 1983). One reason for the interest in predictive inferences is their important relation to what is known about the causal structure of narratives. The processing of narratives is facilitated by the logical ordering of events (Trabasco \& van den Broek, 1985). If readers are going to make an inference to facilitate their assimilation of upcoming text, it seems likely that they would make an inference that is related to the causal coherence of the narrative. However, some researchers have made the claim that people do not make predictive inferences. For example, Haviland and Clark (1974) claimed that backward inferences, which connect text elements, are routinely made, but that forward (predictive) inferences are not.

Two more recent studies of predictive inferences arrived at somewhat different conclusions (McKoon \& Ratcliff, 1986; Potts et al., 1988). McKoon and Ratcliff (1986; see also McKoon \& Ratcliff, 1989b) examined the speed and accuracy of judging whether target words (e.g., dead for the sentence above) were in the texts. There was inhibition in responding (relative to performance on control words) only when the critical target words were preceded by a priming word from the text. McKoon and Ratcliff (1986) interpreted these false recognition data as indicating that predictive inferences are "minimally" drawn. They argued that the target words did not match the encoded text representations sufficiently to yield inhibition when presented alone, but that when a target was combined with a prime from the sentence, there was enough of a match to inhibit responses to the target word. Thus, they suggested that the inference about the actress in the example above would be encoded as "something bad happened."

Potts et al. (1988) claimed that the false recognition procedure used by McKoon and Ratcliff (1986) might result in inference generation at the time of testing rather than during comprehension. Even though McKoon and Ratcliff used a deadline for subjects' responses in order to prevent off-line inferences, Potts et al. argued that a more appropriate procedure would be to use a task that does not involve relating the target back to the priming context (but see McKoon \& Ratcliff, 1989a, for a reply). Potts et al. compared priming in two tasks: lexical decision and naming. They found evidence of priming of predictable concepts only with the lexical decision task. However, the lexical decision task may be subject to a context check between the prime and the target (see, e.g., Forster, 1979; Neely, Keefe, \& Ross, 1989). Because such a context check is less likely to compromise the naming data, Potts et al. concluded that previous evidence that readers make predictive inferences was due to an off-line check between the targets and the preceding context.
The rather conservative view of the comprehension process that emerges from the data obtained by Potts et al. (1988) may underestimate readers' use of predictive inferences. There are two reasons for concern regarding Potts et al.'s conclusion. The first is that the materials used in their study may represent an impoverished context for inference generation. The second has to do with the naming task as a means of detecting inference use. We will elaborate on each of these points in turn.

\section{Foregrounded Versus Backgrounded \\ Inference Contexts}

Writers use several techniques to emphasize particular concepts. For example, a term that serves as a sentence topic, or a term to which a pronominal reference is made, can become the focus of processing (Chafe, 1972; Sanford \& Garrod, 1981). Such terms are said to be foregrounded. The special status afforded foregrounded terms is in part due to their increased likelihood of being connected with later elements of text. For example, terms in the foreground are the ones most likely to be evaluated as the possible referents of a later noun or pronoun (e.g., Fletcher, 1984; Glenberg, Meyer, \& Lindem, 1987). Thus, foregrounded terms are especially important for coherence.

If readers generate elaborative inferences to promote coherence, foregrounding may have an important controlling influence over when elaborative inferences are made (Whitney, 1986). No one would argue that readers generate all possible elaborations of even simple texts, because there is virtually no end to the number of possible elaborations (Reiger, 1975). If elaborations are going to be made, they are more likely to be related to foregrounded concepts, because these concepts are likely to be repeated and become part of the overall representation of the text (Sanford \& Garrod, 1981).

In the case of predictive inferences, it seems unlikely that readers would make inferences about the consequences of an action involving a concept that is not in the foreground. However, an action involving a foregrounded concept might lead to a prediction about what is likely to happen next in the text.

Our examination of the $\mathbf{4 0}$ "inference situations" used by Potts et al. (1988) revealed that, in most cases, the key term related to the target inference was not the focus of the passage. In their study, each situation was described in a two-sentence passage. Three prime versions of each passage were constructed: predicting, coherence, and control. As in McKoon and Ratcliff's (1986) study, the predicting prime contained information that might lead the reader to predict the consequence of an action. For example, the word "broke" might be primed by the following passage:

No longer able to control his anger, the husband threw the delicate porcelain vase against the wall. He had been feeling angry for weeks, but had refused to seek help.

For the coherence version, the last sentence of the para- 
graph required the inference in order for the passage to make sense. For example, the second sentence in the above paragraph was changed to: "It cost him well over one hundred dollars to replace the vase."

The control version used the same key words, but in such a way that the inference test word was not implied:

In one final attempt to win the delicate porcelain vase, the angry husband threw the ball at the bowling pins that stood against the wall. He had never won anything and was determined not to miss this time.

This version controlled for the possibility that word association, rather than the predictive nature of the passage, might activate the inference test word.

In the example of the predictive passage given above, the topic of the passage is the man and his anger, not the delicate vase. This is clear from the second sentence, which mentions the anger of the man and makes no reference to the vase that was thrown. Consider another priming passage from the study by Potts et al. (1988):

The director and cameraman were preparing to shoot closeups of the actress on the edge of the roof of the 14th story building when suddenly the actress fell. The director was talking to the cameraman and did not see what happened.

Unlike in the previous example, there is an anaphoric reference ("what happened") in the second sentence that foregrounds the key action in the first sentence, which was related to the predictive inference.

In reviewing the materials used in the Potts et al. (1988) study, we found that in 28 of the passages the second sentence made no anaphoric reference to the terms involved in the potential predictive inference. In the other 12 passages, the second sentence made reference to the terms related to the inference. In the present study, we created foregrounded and backgrounded versions of each predicting passage by manipulating the presence or absence of such anaphoric references.

\section{Detecting Predictive Inferences}

Of course, even if readers do make predictive inferences in the foregrounded contexts, we cannot find evidence for the inferences unless the task used to detect the inferences is sensitive to inference generation. Potts et al. (1988) used naming times to detect inferences because of the insensitivity of this task to context checking. However, it is also clear from their data that the naming task is a relatively insensitive index of semantic priming even when the inference must be made to achieve coherence. In one experiment with naming times, Potts et al. failed to find a significant difference between the coherence and control conditions. In another experiment, the effects were marginal. Only when the results of the two experiments were combined did priming in the coherence condition reach significance for both subjects and items. Therefore, even when the context was such that subjects were expected to consistently make the appropriate bridging inference, it was difficult to detect the inferences with the naming task.
Data obtained by Lucas, Tanenhaus, and Carlson (1990) shed light on why the naming tasks may be insensitive to elaborative inferences. Lucas et al. compared the lexical decision task and the naming task in terms of how well they detected activation of instrumental inferences and the antecedents of anaphors. They found that lexical decision, but not naming, detected inferences that were part of a discourse model. The naming task was primarily sensitive to activation of entries in the lexicon, as opposed to concepts in the discourse model. In addition, Lucas et al. ran a control experiment in which context checking in lexical decision was ruled out as a factor in their experiments. Thus, concerns over context checking in the lexical decision task that originate from studies of single-word priming (e.g., Forster, 1979) may not generalize to the use of lexical decision to study information primed by sentences.

To summarize, because of problems in materials and methods, recent research on predictive inferences may underestimate their use. To provide a better test of readers' use of predictive inferences, we contrasted foregrounded and backgrounded versions of passages adapted from Potts et al. (1988). Because of the controversy surrounding the appropriate means of detecting inference generation, we employed several different dependent measures, including constrained word-stem (CWS) completion, lexical decision, and naming. The CWS paradigm was recently developed by Whitney and Williams-Whitney (1990). In this paradigm, a word-stem completion task is inserted between the reading of a text and an explicit test of comprehension of the text. Using this task, Whitney and Williams-Whitney (1990) replicated prior Stroop results obtained by Whitney (1986). That is, subjects filled in the constrained stems with target inference words more often after presentation of priming contexts than they did after presentation of control contexts. For example, the stem s h _ _ _ was completed with "shark" more often after "The fish attacked the swimmer" than it was after a control sentence. The CWS task appears to be both sensitive and unobtrusive, because it can be presented as a "distractor task" between reading and a test of comprehension. In the first experiment, we used the CWS task to detect the use of predictive inferences in foregrounded, backgrounded, and coherence versions of passages adapted from the set used by Potts et al. (1988).

\section{EXPERIMENT 1}

One of the positive features of the CWS task is that it appears to be quite sensitive to semantic activation, but does not involve a binary decision as does the lexical decision task. Forster (1979) suggested that because the lexical decision task requires a binary decision it is more subject than naming to a postaccess context-checking procedure (however, cf. Lucas et al., 1990). Briefly, the notion is that a check for semantic congruity with a preceding context can serve as a source of evidence for the "yes" or "no" response. This is less likely to occur when what must be accessed is the procedural knowledge required 
in order to generate a word. In such a case, a postaccess match with the preceding context will not be very helpful to the required response.

The CWS task is like the naming task in that there is no binary decision. The subject must select and name a word that fits the constraints provided by the target. The selection of a particular word candidate may be biased by prior semantic activation, but a postaccess context check would not be particularly helpful in making the required response (as long as filler trials are used to avoid having the subjects adopt guessing strategies). Unlike in the naming task, however, the processing of the target in the CWS task is not so automatic and data driven as to override the effects of prior semantic activation. ${ }^{1}$ We predicted that we would see evidence for predictive inferences with the CWS task, but only with foregrounded passages.

\section{Method}

Subjects and Design. The subjects were $\mathbf{4 0}$ undergraduates who participated in partial fulfillment of a requirement in introductory psychology. All of the subjects were native speakers of English. The single independent variable, type of prime, was manipulated within subjects and had four levels: coherence, foregrounded, backgrounded, and control.

Materials. Sixteen passages were adapted from the materials used by Potts et al. (1988). Of the 16 passages used, the predicting version was judged as foregrounded in 9 cases and as backgrounded in 7 cases. An alternate version was written for each passage so that the same action appeared in a foregrounded and a backgrounded version. The control versions of each passage were modified to incorporate key words from the new versions of the predicting passages used here. One other modification of the passages was made: To the beginning of each passage, we added two sentences that provided some setting information so that the materials would seem more natural. These sentences also provided additional material from which comprehension questions could be drawn. A sample set of passages is shown in Table 1 .

In the example in the table, the first passage is considered foregrounded because the last sentence makes a reference to the bees,

Table 1

Sample Passage Set Used in Experiment 1

\section{Foregrounded}

Joan was enjoying her new summer job. She took a deep breath of fresh air before beginning her chores. The angry swarm of bees flew out of the hive and landed on Joan's hand. The bees had recently been moved into a new hive during Joan's day off.

\section{Backgrounded}

Joan was enjoying her new summer job. She took a deep breath of fresh air before beginning her chores. The angry swarm of bees flew out of the hive and landed on Joan's hand. Joan's job was to see that the large apple orchard was pollinated.

\section{Coherence}

Joan was enjoying her new summer job. She took a deep breath of fresh air before beginning her chores. The angry swarm of bees flew out of the hive and landed on Joan's hand. Her hand was soon very sore and she regretted having bothered the bees.

\section{Control}

Joan was angry with her husband for not finishing his orchard chores. The bee swarm had not been moved to their new hive. The old hive was too near where the duster landed in the summer.

Target CWS: $\mathbf{s}$ t ___(Sting) which are critical to the predictive inference. The last sentence of the backgrounded passage refers to Joan but has no reference to the bees mentioned in the previous sentence.

The CWSs for each passage were created by using the rule in Whitney and Williams-Whitney's (1990) study. One letter was provided for words with four or fewer letters, and two letters were provided for words with five or more letters. However, for a few of the targets, providing the first two letters yielded identical stems for different targets (e.g., "sting" and "study"). In these cases, we provided three letters for the CWS target. A pilot study on 20 subjects showed that all of the targets, including the ones with three letters, had baseline completion rates below $40 \%$. The average baseline completion rate was $10 \%$

Another pilot study was conducted to ensure that the foregrounded, backgrounded, and coherence versions of each passage suggested the target inference. Three lists were created, each containing one of the three prime versions of each passage. The passages were given to 39 subjects, who answered a question after each one. The questions were designed to elicit the target inferences. For example, a passage about a girl who "saw the police car turn on its flashing red lights and wave her car over to the side of the road" was followed by the question, "What happened to the girl when she was pulled over?" The subjects responded with answers such as, "She got a ticket." For this passage, "ticket" was the target word. The different versions of each passage were quite uniform in eliciting the appropriate inference term, and across all passages, the target word was given $86 \%$ of the time.

We used filler trials and filler CWSs to disguise the true nature of the task. For the filler trials, we added sentences about setting to 20 passages from Potts et al. (1988) that were not used as critical trials. Two CWSs were used on each trial, but only the first was the target of interest.

Procedure. With filler passages included, each subject was presented with 36 passages, which included 16 critical trials (4 of each prime type). Each subject was given one of four stimulus lists. Each list contained one version from each set of passages, and each version appeared equally often across lists. The trials were separated into 3 blocks of 12. Care was taken in assigning passages to blocks so that a passage did not appear in the same block as another passage containing a word that would fit into the first passage's CWS. Three practice trials (constructed as the filler trials) were given at the beginning of the experiment. The order of blocks was randomized for each subject.

The experiment was presented as a study examining the subjects' ability to remember the gist of what they had read after a brief distractor task. The materials were presented on a color monitor controlled by a microcomputer. The subjects controlled the presentation of the passages with a keypress. After reading a passage, each subject pressed a key and the first CWS appeared on the screen for $7 \mathrm{sec}$. It was then replaced by the second CWS, which also remained present for $7 \mathrm{sec}$. For each CWS, the subjects were instructed to say aloud the first word that came to mind that fit the blanks. The experimenter recorded all responses to the CWS targets. The second CWS was followed by a true/false question about the passage. Each question required simple factual knowledge of the sentence. Subjects pressed one of two keys to record their answers and were given immediate feedback on the screen as to whether or not their answers were correct.

Subjects were run individually in a single session lasting approximately $\mathbf{3 0} \mathrm{min}$. Subjects were given a brief rest after each block of 12 trials.

\section{Results and Discussion}

Comprehension questions. Two subjects were replaced because of poor performance on the comprehension questions. All other subjects scored above $75 \%$. The mean performance was approximately $90 \%$ correct. 
Target completion. The mean proportions of targets completed were analyzed with a repeated measures analysis of variance (ANOVA). The analyses were performed on the raw data and on arc-sine transformations of the data. The pattern of results was the same for both sets of analyses, so we report only the untransformed data analyses. All effects reported as significant reached at least the .05 alpha level.

The mean proportions of targets completed with the intended words were .31 (coherence), .29 (foregrounded), .21 (backgrounded), and .16 (control). The effect of type of prime was significant for subjects $[F(3,117)=4.44$, $\left.M S_{\mathrm{e}}=.05\right]$ and for items $\left[F(3,45)=4.97, M S_{\mathrm{e}}=.01\right]$. For both subjects and items, $t$ tests based on the $M S_{e}$ term showed that the means of both the coherence and foregrounded conditions were greater than those of the control, but those of the backgrounded condition were not different from those of the control.

We also performed a direct test of whether the major manipulation of the experiment, foregrounded versus backgrounded passages, resulted in different levels of priming. The priming levels ( $13 \%$ vs. $5 \%)$ were significantly different $[t(39)=2.16]$.

These data support the hypothesis that actions involving foregrounded topics lead to inferences about the likely consequences of the actions. The methods and materials used in previous studies, which have yielded little or no evidence for predictive inferences, appear to underestimate inference use.

The findings of Experiment 1 suggest an alternative interpretation of the data obtained by Potts et al. (1988). They found evidence for predictive inferences with lexical decision but not with naming tasks. Because of the presumed susceptibility of lexical decision to context checking, Potts et al. argued that the naming data yielded the more valid results. However, Lucas et al. (1990) found that lexical decision times, as used in research to study on-line inference generation, are not affected by context checks. Because the materials used by Potts et al. foregrounded the concept relevant to the inference only $30 \%$ of the time, it seems likely that only the lexical decision task was sensitive enough to detect the predictive inferences in their study. In the next set of experiments, we attempted to provide converging evidence for this conclusion by using lexical decision and naming tasks to test for predictive inferences.

\section{GENERAL METHODOLOGY FOR EXPERIMENTS 2 AND 3}

We felt it was important to keep the stimulus materials the same as in the first experiment so that the results could be compared, but this presented a problem. Reaction time tasks require more trials per condition than does the CWS task in order to attain stable means. Consequently, we could not include all four levels of the prime variable in a single within-subjects design. Instead, we ran a series of experiments in which activation of target concepts was assessed after the presentation of one type of predictive inference passage and after the presentation of control passages.

Although Potts et al. (1988) did not use filler trials when they tested for predictive inferences with lexical decision and naming, we interspersed 16 filler trials among the 16 critical trials in each of our experiments. Stimulus presentation and timing were controlled by an IBM PC interfaced with a John Bell Universal I/O board and millisecond clock.

Each experiment had 36 subjects, drawn from the same pool as the first experiment. Subjects who failed to answer $75 \%$ of the comprehension questions correctly were replaced. Reaction times greater than two standard deviations above each subject's mean were excluded as outliers. Less than $2 \%$ of the data in each experiment were excluded. We do not present error data individually by experiment because, as is typical of such lexical decision and naming experiments, the error rates were low (5\% or less) and uniform across conditions.

\section{EXPERIMENT 2 \\ Lexical Decision}

This series of experiments was designed to determine whether converging evidence for the CWS results could be obtained with lexical decision. If the results of the first experiment are valid, and if the lexical decision task detects on-line inference generation, we should find evidence for predictive inferences with foregrounded passages but not with backgrounded passages. Furthermore, when passages are used that preserve the general context but do not suggest the inference, we should nót find evidence for activation of the target concepts. This manipulation was used to assess whether context checking could account for the lexical decision results (cf. Lucas et al., 1990).

\section{Experiment 2A}

\section{Method}

The foregrounded and control passages were used in this experiment. Two versions of the experiment were created. In each version, half of the critical trials were priming trials and half were controls. Across versions, each passage appeared in each of the two conditions. The order of passages was randomized within each list.

The subjects read each passage at their own pace. After the subjects pressed a key to remove the passage, there was a 500 -msec delay before the target appeared. The subjects indicated that they perceived it as a word by pressing the " 1 " key and as a nonword by pressing the $2 \mathrm{key}$. The keyboard had an average delay of $9 \mathrm{msec}$. Speed and accuracy of responding were stressed equally in the instructions to the subjects. All trials ended with a comprehension question about the passage. Each subject was given eight practice trials, and each appeared to be comfortable with the procedure by that point.

The computer automatically recorded reaction times and answers to the comprehension questions. The entire procedure took approximately $20 \mathrm{~min}$ per subject.

\section{Results and Discussion}

Two subjects were replaced for failure to meet the criterion of $75 \%$ on the comprehension questions. The mean 
reaction times (in milliseconds) for the foregrounded and control conditions, respectively, were $715 \mathrm{msec}$ $(S D=116)$ and $743 \mathrm{msec}(S D=171)$. This difference was significant both for subjects $[t(35)=2.13]$ and for items $[t(15)=3.32]$. These data, like the CWS data, suggest that predictive inferences are made in foregrounded contexts. We then tried to determine whether this result would be obtained with the backgrounded passages.

\section{Experiment 2B}

\section{Method}

The method was the same as for Experiment 2A, except that the backgrounded versions of each passage were used. We had to replace 4 subjects who failed to meet the comprehension criterion.

\section{Results}

The change in passages led to a very different outcome in the reaction time data. There was no priming effect in this experiment. The mean reaction times were 712 msec $(S D=147)$ in the background condition and 711 msec $(S D=137)$ in the control condition. Again, the lexical decision data provided converging evidence for the CWS data.

We also conducted a combined test for priming effects across these two experiments. An ANOVA was conducted on the two-factor mixed design, stimulus type (foregrounded vs. backgrounded) $\times$ priming (priming sentence vs. control sentence). The interaction, indicating a greater priming effect in Experiment 2A than in Experiment 2B, was marginally significant $\left[F(1,70)=2.88, M S_{e}=\right.$ $2,877, p=.09]$. Of course, this analysis has somewhat less power than the previous comparisons, because the foreground-background manipulation occurred between subjects. Overall, the lexical decision data, like the CWS data, support the hypothesis that foregrounding increases the likelihood of a predictive inference.

\section{Experiment 2C}

In this experiment, we tested whether a simple contextchecking explanation could account for the finding that the target terms were activated in the foregrounded condition. We thought this unlikely for two reasons: (1) the same results were obtained with the CWS task, which does not involve a binary decision, and (2) the weak evidence for priming the backgrounded condition does not support the context-checking argument. Nevertheless, we thought it useful to test for activation of the targets when the critical noun in the passage was foregrounded, but no inference was suggested (cf. Lucas et al., 1990).

\footnotetext{
Method

The foregrounded passages from the first experiment were rewritten so that the key noun that participated in the inference remained foregrounded, but no inference was suggested. For example, we changed the third sentence of the foregrounded prime shown in Table 1 to read, "The angry swarm of bees flew out of the hive close to Joan's hand." Slight adjustments were made to the corresponding control primes to accommodate any new content words introduced into the primes. All other aspects of the experiment replicated Experiement 2A.
}

\section{Results and Discussion}

Two subjects were replaced for failure to meet the comprehension criterion. There was no evidence for priming effects in the reaction time data. The mean reaction time (in milliseconds) in the context condition was $738 \mathrm{msec}$ $(S D=142)$, compared with $735 \mathrm{msec}(S D=138)$ in the control condition. Obviously, this difference did not approach significance.

Like Lucas et al. (1990), we found no evidence that the lexical decision data were compromised by a simple context-checking strategy. One could imagine a more sophisticated strategy that would be consistent with the present results; subjects could base their responses on the congruity of the target and an integrated model of the prime. However, this version of context checking would seem to require that the inference in question be part of the representation of the prime. Any more superficial context check should have yielded priming in this experiment.

\section{EXPERIMENT 3 Naming}

The lexical decision data provided converging evidence for the hypothesis that predictive inferences are made in foregrounded contexts. We argued earlier that prior results indicating that predictive inferences are not made were due in part to the frequent use of backgrounded passages. However, we also hypothesized that the naming task was too insensitive to detect the use of predictive inferences. We tested this claim by examining target naming following the foregrounded and coherence passages in comparison with the control passages.

\section{Method}

\section{Experiment 3A}

The design was identical to that of Experiment 2A, except that the naming task was used to test for priming in the foregrounded passages. A microphone and a voice-operated relay (Gerbrands Model 1341T) were interfaced with the computer to allow collection of naming times.

\section{Results and Discussion}

One subject was replaced for failing to meet the comprehension criterion. The mean naming time for both the foregrounded condition and the control conditions was $549 \mathrm{msec}$ (SDs $=86$ and 90 , respectively, for the foregrounded and control conditions). Thus, as expected, no evidence for priming was found with the naming task. Next, we tested whether this lack of priming was due to insensitivity of the task or to a true lack of inference generation.

\section{Experiment 3B}

\section{Method}

The coherence and control versions of each passage were used for this experiment. All other aspects of the design were the same as in the previous experiment.

\section{Results and Discussion}

Two subjects were replaced for failing to meet the comprehension criterion. The mean naming times were 
$546 \mathrm{msec}(S D=73)$ and $556 \mathrm{msec}(S D=89)$ for coherence and control, respectively. These data are almost identical to those obtained in Potts et al.'s (1988) Experiment 3. They found an 11-msec difference between coherence and control, and we obtained a $10-\mathrm{msec}$ difference. Just as Potts et al. found, the effect was marginal for subjects $[t(15)=1.65, p=.10]$, but not for items $[t(15)=.88, p>.30]$. With greater power, small but significant priming effects can be obtained in the coherence condition (see Potts et al., Experiment 4). This result suggests to us that the naming task is much less sensitive to information primed as a result of an inference than is the CWS task or the lexical decision task.

\section{CONCLUSIONS AND DIRECTIONS FOR FUTURE RESEARCH}

We began this work by noting that the extant views on elaborative inferences are quite polarized. The present study supports neither the view that readers are extremely conservative about making elaborative inferences nor the view that top-down influences predominate in reading. Instead, the use of elaborative inferences appears to be context sensitive (cf. McKoon \& Ratcliff, 1989b; Whitney \& Williams-Whitney, 1990). As predicted, the first experiment showed that foregrounding is a potent controlling factor. The other experiments, the results of which are summarized in Figure 1, provide: (1) converging evidence that foregrounding is a critical factor in controlling inference use, and (2) support for the hypothesis that the naming task is insensitive to inference generation (see also Lucas et al., 1990).

Although it is clear that foregrounding was important to inference use in this study, the nature of the manipulation that we used suggests an interesting question that merits further study. Recall that the difference between the foregrounded and backgrounded passages occurred in the fourth and final sentence. This manipulation emerged naturally from our inspection of the materials used in pre-

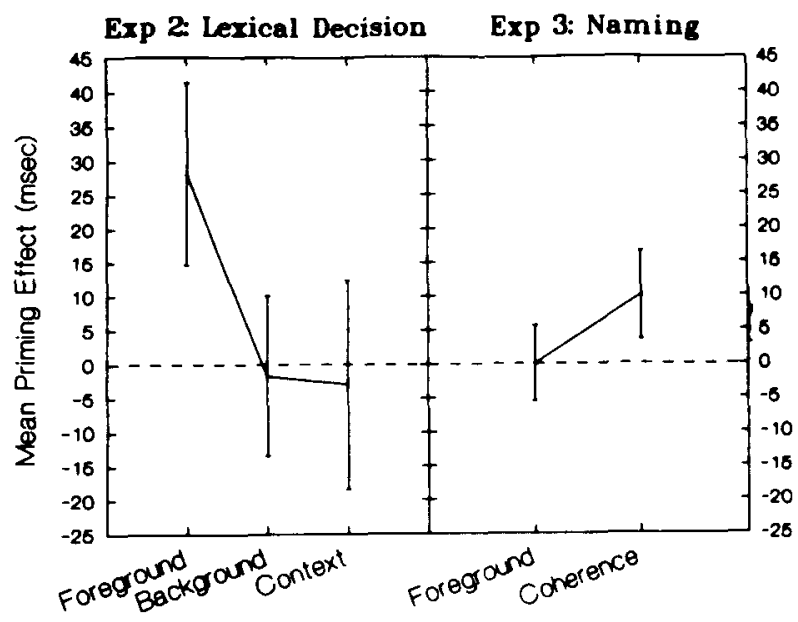

Figure 1. Priming effects (means and standard errors) across Experiments 2 and 3. vious studies. Foregrounding of the term involved in the critical action in the last sentence appears to influence the topic of the passage as a whole, and it influenced inference use in the way that we predicted. However, our manipulation of foregrounding raises a question concerning the locus of the inference during comprehension. When the subjects read the foregrounded passages, in which sentences did they make the inference?

One possibility is that the predictive inference was generated as subjects read the third sentence, in which the critical action took place. If the fourth sentence made an anaphoric reference relevant to the prediction, the activation of the inference was maintained. If the fourth sentence shifted the emphasis away from the terms involved in the key action, activation of the consequences of that action were inhibited or passively decayed. According to this "activation-deactivation" model, the predictive inferences are actually made in both foregrounded and backgrounded contexts, but we detect the inferences only in the former case.

Another possibility is that the third sentence only weakly or partially primed inference-relevant concepts (cf. McKoon \& Ratcliff, 1989b). The inference is then completed only if the fourth sentence provides contextual support in terms of evidence that the key term involved in the inference is an important text element. According to this "strength of encoding" model, the inference occurs in stages as processing spills over from one sentence to the next.

The present study was not designed to test between the activation-deactivation and the strength of encoding models. However, it should be possible to test explicitly for the locus of the inference in future studies if on-line techniques for studying activation of word meanings (see, e.g., Kellas, Paul, Martin, \& Simpson, 1991) are imported into the inference literature. It is important to note that tests of whether critical sentences (such as the third sentences in this study) activate the inferences when presented in isolation are not sufficient to test the two models. It seems likely that single sentences represent a very impoverished context for studying inferences that may participate in text-integration processes.

Finally, a major issue in this area of research has been the choice of methods for studying elaborative inferences (see Keenan, Golding, Potts, Jennings, \& Aman, 1990; McKoon \& Ratcliff, 1989a). As noted earlier, Potts et al. (1988) criticized the item-recognition procedure of McKoon and Ratcliff for its possible susceptibility to context checking. McKoon and Ratcliff (1989a) defended their procedure and criticized the naming task used by Potts et al. (1988). Whitney and Waring (1989) criticized the item-recognition procedure for a different reasonthat the procedure orients readers to the surface form of what they read and not to any deep level of comprehension.

Although the present study does not settle all of the methodological issues surrounding the study of elaboration in comprehension, we believe that data shed some light on the problem. Taken together with the data 
obtained by Lucas et al. (1990), the present data suggest that the naming task is not well suited for determining what information is inferred to be part of the mental model of a text. In contrast, the CWS task and the lexical decision task are sensitive to inference generation. Moreover, the two tasks provide useful converging evidence for each other because they rely on different decision processes.

Given the current methodological controversies, it seems best to provide converging evidence for inference use. However, although eclecticism in our techniques seems necessary, it is not sufficient. If we are to understand the role of elaborative inferences in comprehension, we must examine the pattern of the use (and nonuse) of elaborative inferences in a variety of contexts. Several classes of contextual variables that merit consideration in future research include the role played by critical terms in the text (e.g., foregrounding), the level to which readers are oriented (e.g., surface form or gist representation), and individual differences (e.g., reading ability; see Whitney \& Williams-Whitney, 1990).

We have only begun to explore the contextual factors that control inference use, but, intuitively, it would appear to be a fruitful course of investigation. Some reading theorists have been willing to make the unqualified claim that readers do not make forward, or predictive, inferences. Such blanket statements notwithstanding, who could read an Agatha Christie novel without making predictive inferences? We cannot claim to understand the role of elaboration in comprehension until we understand the conditions in which particular elaborative inferences are used, as well as the conditions in which such inferences are not used.

\section{REFERENCES}

Anderson, R. C., Pichert, J. W., Goetz, E. T., Schallert, D. L., SteVens, K. W., \& Trollip, S. R. (1976). Instantiation of general terms. Journal of Verbal Learning \& Verbal Behavior, 15, 667-679.

Bransford, J. D., Johnson, M. K. (1973). Consideration of some problems of comprehension. In W. G. Chase (Ed.), Visual information processing (pp. 383-438). New York: Academic Press.

ChafE, W. (1972). Discourse structure and human knowledge. In J. B. Carroll \& R. O. Freedle (Eds.), Language comprehension and the acquisition of knowledge (pp. 41-69). Washington, DC: Winston.

Corbett, A. T., Dosher, B. A. (1978). Instrument inferences in sentence encoding. Joumal of Verbal Learning \& Verbal Behavior, 17, 479-491.

Dosher, B. A., \& CorbetT, A. T. (1982). Instrument inferences and verb schemata. Memory, \& Cognition, 10, 531-539.

DuFF, S. A. (1986). Role of expectations in sentence integration. Journal of Experimental Psychology: Learning, Memory, \& Cognition, 12, 208-219.

Fletcher, C. R. (1984). Markedness and topic continuity in discourse processing. Journal of Verbal Learning \& Verbal Behavior, 23, 487-493.

Forster, K. I. (1979). Levels of processing and the structure of the language processor. In W. E. Cooper \& E. C. T. Walker (Eds.), Sentence processing: Psycholinguistic studies presented to Merrill Garrett (pp. 27-85). Cambridge, MA: MIT Press.

Glenberg, A. M., Meyer, M., \& Lindem, K. (1987). Mental models contribute to foregrounding during text comprehension. Joumal of Memory \& Language, 26, 69-83.

Graesser, A. C., C Clark, L. F. (1985). Structures and procedures of implicit knowledge. Norwood, NJ: Ablex.

Haviland, S. E., Clark, H. H. (1974). What's new? Acquiring new information as a process in comprehension. Joumal of Vertal Learning \& Verbal Behavior, 13, 512-521.

Johnson-Laird, P. N. (1983). Mental models. Cambridge, MA: Harvard University Press.

Keenan, J. M., Golding, J. M., Potts, G. R., Jennings, T. M., AMAN, C. J. (1990). Methodological issues in evaluating the occurrence of inferences. In A. C. Graesser \& G. H. Bower (Eds.), Inferences and text comprehension (pp. 295-312). New York: Academic Press.

Kellas, G., Paul, S. T., Martin, M., a Simpson, G. B. (1991). Contextual feature activation and meaning access. In G. B. Simpson (Ed.), Understanding word and sentence (pp. 47-72). Amsterdam: NorthHolland.

lucas, M. M., Tanenhaus, M. K., Carlson, G. N. (1990). Levels of representation in the interpretation of anaphoric reference and instrument inference. Memory \& Cognition, 18, 611-631.

McKoon, G., RATCLIF, R. (1986). Inferences about predictable events. Journal of Experimental Psychology: Leaming, Memory, \& Cognition, 12, 82-91.

McKoON, G., RATCLIF, R. (1989a). Assessing the occurrence of elaborative inference with recognition: Compatibility checking vs. compound cue theory. Journal of Memory \& Language, 28, 547-563.

McKoon, G., RATCLFF, R. (1989b). Semantic associations and elaborative inference. Journal of Experimental Psychology: Learning, Memory, \& Cognition, 15, 326-338.

Neely, J. H., KeEFe D. E., \& Ross, K. (1989). Semantic priming in the lexical decision task: Roles of prospective prime-generated expectancies and retrospective semantic matching. Joumal of Experimental Psychology: Learning, Memory, \& Cognition, 15, 1003-1019.

PARIs, S. G., Lindauer, B. K. (1976). The role of inference in children's comprehension and memory for stories. Cognitive Psychology, 8, 217-227.

Potts, G. R., Keenan, J. M., Golding, G. M. (1988). Assessing the occurrence of elaborative inferences: Lexical decision versus naming. Journal of Memory \& Language, 27, 399-415.

Rayner, K., Pollatsek, A. (1989). The psychology of reading. Englewood Cliffs, NJ: Prentice-Hall.

ReIGER, C. J., III. (1975). Conceptual memory and inferences. In R. C. Schank (Ed.), Conceptual information processing (pp. 157-288). Amsterdam: North-Holland.

SANFORD, A. J., \& GARROD, S. C. (1981). Understanding written language. Chichester, U.K.: Wiley.

Schank, R. C. (1978). Predictive understanding. In R. N. Campbell \& T. Smith (Eds.), Recent advances in the psychology of language: Formal and experimental approaches (pp. 91-101). New York: Plenum.

Seifert, C. M., Robertson, S. P., \& Black, F. B. (1985). Types of inferences generated during reading. Joumal of Memory \& Language, 24, 405-422.

Singer, M., Ferreira, F. (1983). Inferring consequences in story comprehension. Journal of Verbal Learning \& Verbal Behavior, 22, 437-448.

Trabasso, T., \& van den Broek, P. W. (1985). Causal relatedness and importance of story events. Journal of Memory \& Language, 24, 595-611.

WhITNEY, P. (1986). Processing category terms in context: Instantiations as inferences. Memory \& Cognition, 14, 39-48.

WhITNEY, P. (1987). Psychological theories of elaborative inferences: Implications for schema-theoretic views of comprehension. Reading Research Quanerly, 22, 299-310.

Whitney, P., WARING, D. A. (1989, November). Task effects on reader's use of elaborative inferences. Paper presented at the meeting of the Psychonomic Society, Atlanta, GA.

WhitNey, P., \& Williams-WhitNey, D., (1990). Toward a contextu- 
alist view of elaborative inferences. In A. C. Graesser \& G. H. Bower (Eds.), The psychology of learning and motivation (pp. 279-293). New York: Academic Press.

\section{NOTE}

1. In a methodological study of the CWS task, Whitney and Waring (1989) found that the task was sensitive only to priming of forward associates. One source of evidence for context checking is priming by backward associates (see Neely, Keefe, \& Ross, 1989). However, no such backward priming has been observed with the CWS task. In addition Whitney and Waring (1989) tested whether subjects developed a conscious strategy of using the primes to complete the targets. Under conditions like those in the present experiment (presenting the CWS task as a distractor, using two targets, and diluting the critical primes among an equal number of fillers) there was no evidence of any influence of subject strategies.

(Manuscript received July 16, 1990;

revision accepted for publication January 9, 1992.)

\section{Members of Underrepresented Groups: Reviewers for Journal Manuscripts Wanted}

On behalf of Memory \& Cognition and Psychonomic Society Publications, I invite you to contact me if you are interested in reviewing manuscripts for Memory \& Cognition. Please send a letter and a copy of your curriculum vita to me at the following address: Memory \& Cognition, Department of Psychology, Indiana University, Bloomington, Indiana 47405. The letter or the vita should contain your complete address (including an electronic mail address if one is available), telephone number, and area(s) of expertise. Our reviewers have published articles in peer-reviewed journals, a standard prerequisite for being selected as a reviewer.

Please note that reviewing manuscripts takes time and must be completed quickly. If you are asked to review a manuscript, you will be expected to provide a thorough and prompt review.

Margaret Jean Intons-Peterson Editor 